\title{
Compressive Sampling of EEG Signals with Finite Rate of Innovation
}

\author{
Kok-Kiong Poh and Pina Marziliano \\ Division of Information Engineering, School of Electrical and Electronic Engineering, Nanyang Technological University, \\ 50 Nanyang Avenue, Singapore 639798 \\ Correspondence should be addressed to Kok-Kiong Poh, pohk0005@ntu.edu.sg
}

Received 30 July 2009; Revised 26 November 2009; Accepted 3 February 2010

Academic Editor: Tan Lee

Copyright ( $\odot 2010$ K.-K. Poh and P. Marziliano. This is an open access article distributed under the Creative Commons Attribution License, which permits unrestricted use, distribution, and reproduction in any medium, provided the original work is properly cited.

\begin{abstract}
Analyses of electroencephalographic signals and subsequent diagnoses can only be done effectively on long term recordings that preserve the signals' morphologies. Currently, electroencephalographic signals are obtained at Nyquist rate or higher, thus introducing redundancies. Existing compression methods remove these redundancies, thereby achieving compression. We propose an alternative compression scheme based on a sampling theory developed for signals with a finite rate of innovation (FRI) which compresses electroencephalographic signals during acquisition. We model the signals as FRI signals and then sample them at their rate of innovation. The signals are thus effectively represented by a small set of Fourier coefficients corresponding to the signals' rate of innovation. Using the FRI theory, original signals can be reconstructed using this set of coefficients. Seventy-two hours of electroencephalographic recording are tested and results based on metrices used in compression literature and morphological similarities of electroencephalographic signals are presented. The proposed method achieves results comparable to that of wavelet compression methods, achieving low reconstruction errors while preserving the morphologiies of the signals. More importantly, it introduces a new framework to acquire electroencephalographic signals at their rate of innovation, thus entailing a less costly low-rate sampling device that does not waste precious computational resources.
\end{abstract}

\section{Introduction}

The electroencephalogram (EEG) is a recording of the brain's neural activities. Since its discovery by Berger [1], many research activities have focussed on how to automatically extract useful information about the brain's conditions based on the distinct characteristics of these electrical signals. Valuable information about the human brain conveyed by the EEG is used in various studies like the nervous system, sleep disorders, epilepsy, and dementia [2]. These applications require acquisition, storage, and automatic processing of EEG during an extended period of time. For example, 24hour monitoring of a multiple-channel EEG is needed for epilepsy patients. Traditionally, the EEG has been bandlimited to the frequency range between 0.1 and $100 \mathrm{~Hz}$; thus a minimum Nyquist sampling rate of $200 \mathrm{~Hz}$ is needed. At the quantization level of 16 bit/sample, a 10-channel EEG for a 24 -hour period would amount to 346 megabytes. Hence, to efficiently store and transmit a huge amount of data, effective compression techniques are desired. While lossy techniques yield higher compression, because of reliability considerations, lossy data compression techniques are not used as the morphology of the signals which are not always well retained. Excellent surveys of the performance of lossless and lossy EEG compression techniques can be found in [3] to [4]. Antoniol and Tonella presented and discussed several classical lossless EEG signal compression methods such as Huffman coding, predictive compression, and transform compression [3]. In [5], Memon et al. discussed lossless compression techniques ranging from simple dictionary searches to sophisticated context modeling. A long-term EEG compression method using features obtained from the signals' power spectral density was proposed in [6] while multi-channel EEG signals were compressed by exploiting the intercorrelation among the EEG channels through the Karhunen-Loeve transform in [7]. Nielsen et al. proposed a signal-dependent wavelet compression scheme that adapted optimal wavelets to biomedical signals for compression [8]. A near-lossless 
compression method described in [9] compressed EEG signals using neural network predictors followed by nonuniform quantization. More recently, a new compression method based on the construction process of the classified signature and envelop vector sets of the EEG signals [4].

The techniques presented above operated on EEG signals obtained at or above Nyquist rate. This acquisition process leads to a collection of huge amounts of irrelevant data, only to be discarded during the compression stage of the signals. Furthermore, transients, which are common in EEG signals, are not bandlimited. Hence, Shannon's sampling theory cannot be applied to sampling EEG signals. Over the last few years, advancements in signal processing and data acquisition introduced a new sampling theory known as compressive sampling or compressed sensing [10]. Aviyente proposed a compressed sensing framework for EEG compression by exploiting the sparsity of EEG signals in a Gabor frame [11]. This method, however, does not operate on the analog EEG signals directly. Compressive sampling, on the other hand, asserts that its acquisition system directly translates analog signals into compressed digital form so that one can recover super-resolved signals from a few measurements [10]. Similarly, we propose to approach the problem of compressing EEG signals at source. In order to address the nonbandlimitedness of the EEG signals, our compression method will be based on the theory of sampling signals with finite rate of innovation (FRI) [12]. This theory has recently been investigated for a compression technique for electrocardiogram (ECG) signals [13] and neonatal EEG seizure signals [14] as well as for EEG seizure source localisation [15].

Our paper is organised as follows. In Section 2, a description of the EEG data, a review on sampling signals with finite rate of innovation, and an FRI model of EEG signals are presented. A scheme for compressively sampling EEG signals with finite rate of innovation will be described in Section 3 . Results and discussions will be presented in Section 4 and finally, a conclusion will summarise our findings and provide directions for our future work.

\section{EEG Data Description and the FRI Model}

2.1. EEG Data Description. A total of 3 sets of normalised EEG signals comprising 72 hours were used for the study. The data is further divided into 10 seconds epochs for processing. All 3 patients experienced similar seizure types at similar locations on the brain. From this dataset, 30 epochs of 10 seconds duration were selected for establishing a finite rate of innovation model of EEG signals while the rest of the data were used to evaluate our compression scheme. The EEG data were acquired using a Neurofile NT digital video EEG system with 128 channels, $256 \mathrm{~Hz}$ sampling rate, and a 16-bit analogue-to-digital converter. Notch or bandpass filters have not been applied. More details of the database can be found in [16]. In our experiments, these EEG signals are assumed to be the source signals. For each patient, there will be 360 epochs and the epochs will be referenced as $P x_{-} y y y$ where $x$ represents the patient number and $y y y$ represents the epoch number.
2.2. Review of Sampling Signals with Finite Rate of Innovation. Consider classes of parametric signals with a finite number of degrees of freedom per unit of time, which is defined as the rate of innovation (e.g., streams of Dirac pulses, nonuniform splines, and piecewise polynomials). It is shown in [12] that although these signals are not bandlimited, they can be sampled uniformly at (or above) the rate of innovation using an appropriate kernel, and then perfectly reconstructed by solving systems of linear equations.

2.2.1. Periodic Stream of Dirac Pulses. Consider a stream of $K$ Dirac pulses periodized with period $\tau, x(t)=\sum_{n \in \mathbb{Z}} c_{n} \delta(t-$ $t_{n}$ ) where $t_{n+K}=t_{n}+\tau$ and $c_{n+K}=c_{n}$, for all $n \in \mathbb{Z}$. This signal has $2 K$ degrees of freedom per period, thus the rate of innovation is

$$
\rho=\frac{2 K}{\tau} .
$$

By taking a continuous-time periodic sinc sampling kernel $h_{B}(t)=B \operatorname{sinc}(B t)$ with bandwidth $B$ greater than or equal to the rate of innovation $\rho$ given by (1), and sampling $y(t)=$ $\left(h_{B} * x\right)(t)$ at $N$ uniform locations $t=n T ; n=0, \ldots, N-$ 1 , where $N \geq 2 M+1, M=\lfloor B \tau / 2\rfloor$ and $M \geq K$, then the samples defined by $y_{n}=\left\langle h_{B}(t-n T), x(t)\right\rangle, n=0,1, \ldots, N-1$ sufficiently represent $x(t)[12]$.

2.2.2. Nonuniform Splines. A signal $x(t)$ is a nonuniform spline of degree $R$ with knots at $\left\{t_{k}\right\}_{k=0}^{K-1}$ if and only if its $(R+1)$ th derivative is a stream of $K$ weighted Dirac pulses $x^{(R+1)}(t)=\sum_{k=0}^{K-1} c_{k} \delta\left(t-t_{k}\right)[17]$. Here, the rate of innovation is $\rho=2 K / \tau$.

Consider a continuous-time periodic nonuniform linear spline $x(t)$ with period $\tau$, containing $K$ pieces of maximum degree $R=1$. By following the sampling method described in Section 2.2.1, $x(t)$ is uniquely defined by $y_{n}=\left\langle h_{B}(t-\right.$ $n T), x(t)\rangle, n=0,1, \ldots, N-1[12]$.

2.2.3. Noisy Case. In this section, we briefly present two types of noise signals that are added to the FRI signals. The first type of noise signal considered is the white noise, which is a zero-mean signal characterised by a flat power spectral density. The second type of noise signal is the $1 / f$ noise whose power spectral density is inversely proportional to its frequency [18]. Accordingly, we define

$$
\begin{aligned}
& S_{w}(f)=N_{w}, \\
& S_{p}(f) \propto \frac{1}{f^{\alpha}},
\end{aligned}
$$

where $S_{w}$ and $S_{p}$ are the power spectral densities of white and $1 / f$ noise, respectively and $0<\alpha<2$.

2.3. Spline-Based FRI Models with Additive Noise. In this section, an FRI model of EEG signals is validated and presented. In particular, we model the EEG signals as

$$
x(t)=x_{s}(t)+n(t),
$$

where $x_{s}(t)$ is the nonuniform spline component, and $n(t)$ is the noise component. We consider the cases of nonuniform 


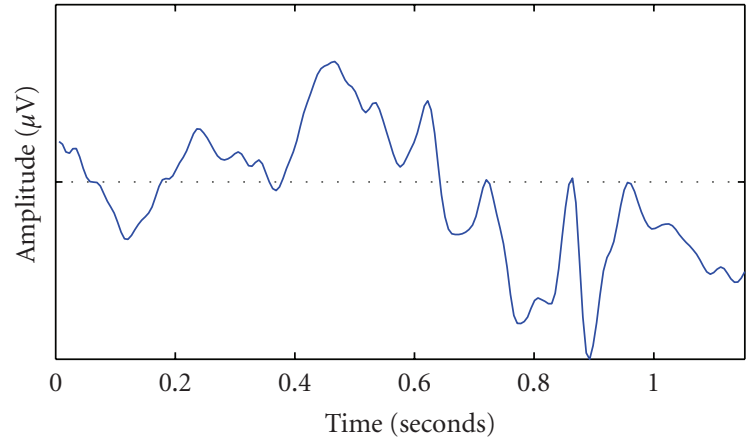

(a)

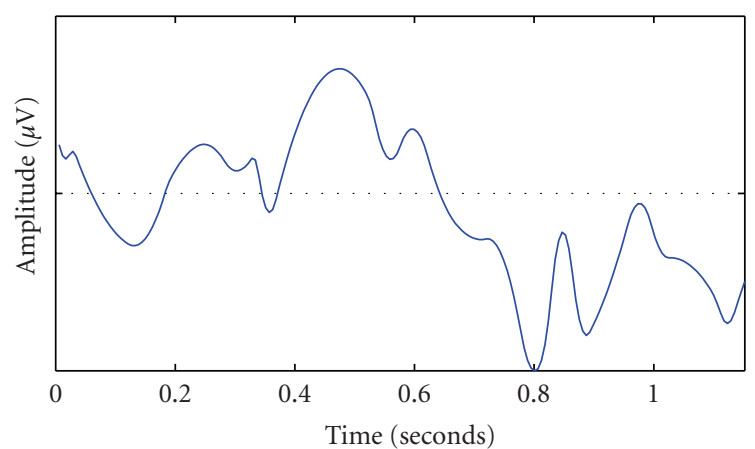

(c)

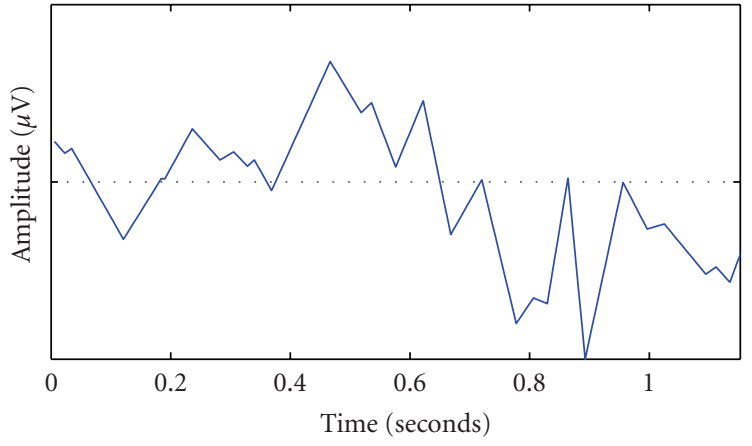

(b)

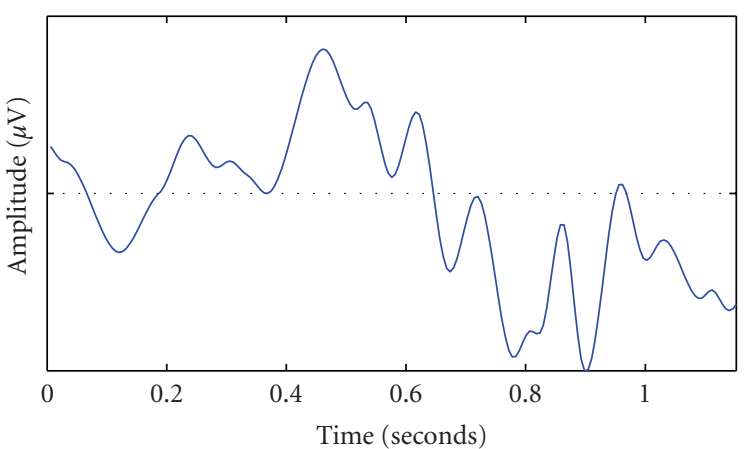

(d)

Figure 1: Comparison of different spline models of EEG. (a) Original EEG signal; (b) Linear spline model, CC $=99.22 \%$, RMSE $=0.0020$, $\mathrm{PRD}=13.07 \%$, MAXERR = 0.0012; (c) Quadratic spline model, CC = 96.00\%, RMSE = 0.0046, PRD = 29.65\%, MAXERR =0.023; (d) Cubic spline model $\mathrm{CC}=99.13 \%$, RMSE $=0.0021, \mathrm{PRD}=13.65 \%$, MAXERR $=0.0011$.

linear spline, nonuniform quadratic spline, and lastly the nonuniform cubic spline (where $R=1,2,3$, respectively in Section 2.2.2) with additive white noise and $1 / f$ noise. We also compare the models with the original signals based on the performance metrics described below and conclude with a suitable FRI model for EEG signals.

2.3.1. Performance Metrics. The following evaluation metrics were employed to determine our method's performance [4].

The compression ratio (CR) is defined as a ratio between the number of bits required to represent the original signal and the compressed signal. First, we define a ratio

$$
C=\frac{b_{\text {orig }}}{b_{\text {comp }}},
$$

where $b_{\text {orig }}$ and $b_{\text {comp }}$ represent the numbers of bits required for the original and compressed signals, respectively.

Thus we can define a CR commonly used in the literature as

$$
\mathrm{CR}(\%)=\frac{C-1}{C} \times 100 .
$$

A metric that can be used to measure distortion is percent root difference (PRD). This metric is commonly used for measuring the distortions in reconstructed biomedical signals such as Electrocardiographic (ECG) signals and EEG signals. For signals of length $J$, PRD can be defined as

$$
\operatorname{PRD}(\%)=\sqrt{\frac{\sum_{i=1}^{J}\left(x_{\text {orig }}(i)-x_{\text {recon }}(i)\right)^{2}}{\sum_{i=1}^{J}\left(x_{\text {orig }}(i)\right)^{2}}} \times 100,
$$

where $x_{\text {orig }}(i)$ and $x_{\text {recon }}(i)$ are the sampled values of the original and reconstructed signals.

Another distortion metric is the root mean square error (RMSE). In data compression, we are interested in finding an optimal approximation for minimizing this metric as defined by the following formula:

$$
\operatorname{RMSE}=\sqrt{\frac{\sum_{i=1}^{J}\left(x_{\text {orig }}(i)-x_{\text {recon }}(i)\right)^{2}}{J}} .
$$

Since the similarity between the reconstructed and original signal is crucial from the clinical point of view, the cross correlation (CC) is used to evaluate the similarity between the original signal and its reconstruction.

$\mathrm{CC}=$

$$
\frac{(1 / J) \sum_{i=1}^{J}\left(x_{\text {orig }}(i)-\overline{x_{\text {orig }}}\right)\left(x_{\text {recon }}(i)-\overline{x_{\text {recon }}}\right)}{\sqrt{(1 / J) \sum_{i=1}^{J}\left(x_{\text {orig }}(i)-\overline{x_{\text {orig }}}\right)^{2}} \sqrt{(1 / J) \sum_{i=1}^{J}\left(x_{\text {recon }}(i)-\overline{x_{\text {recon }}}\right)^{2}}}
$$




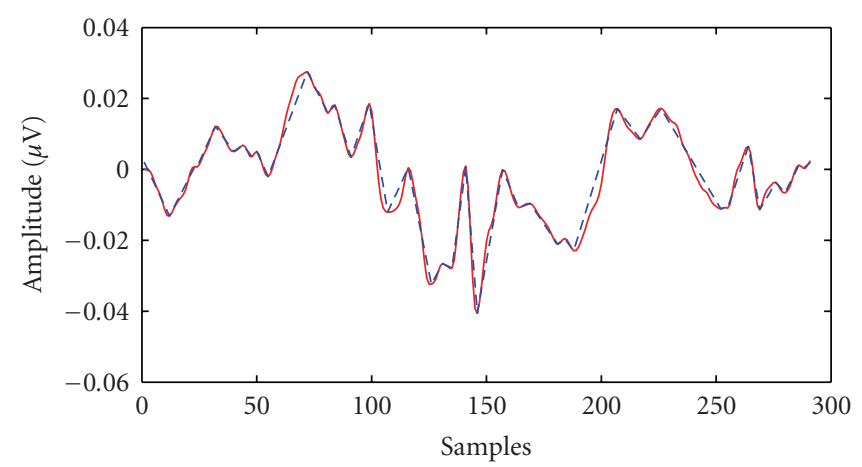

(a)

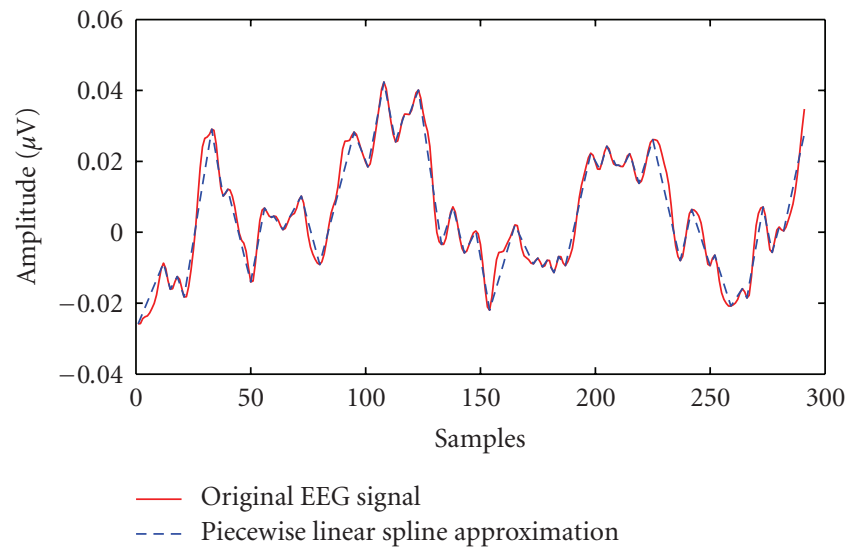

(c)

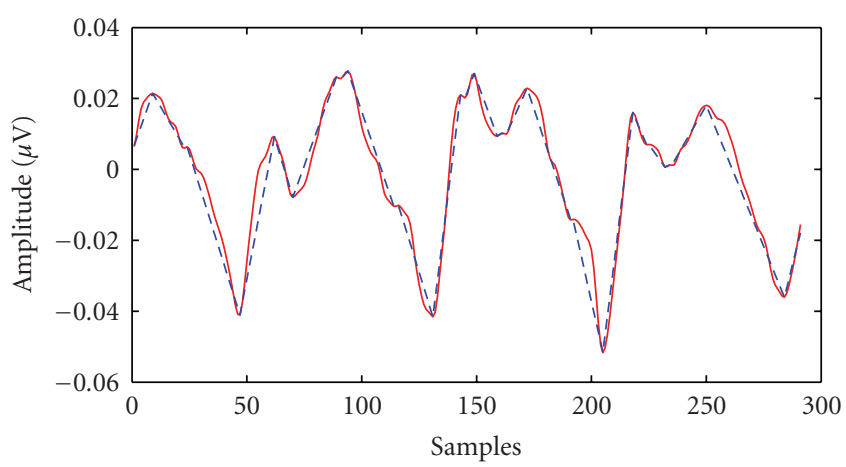

(b)

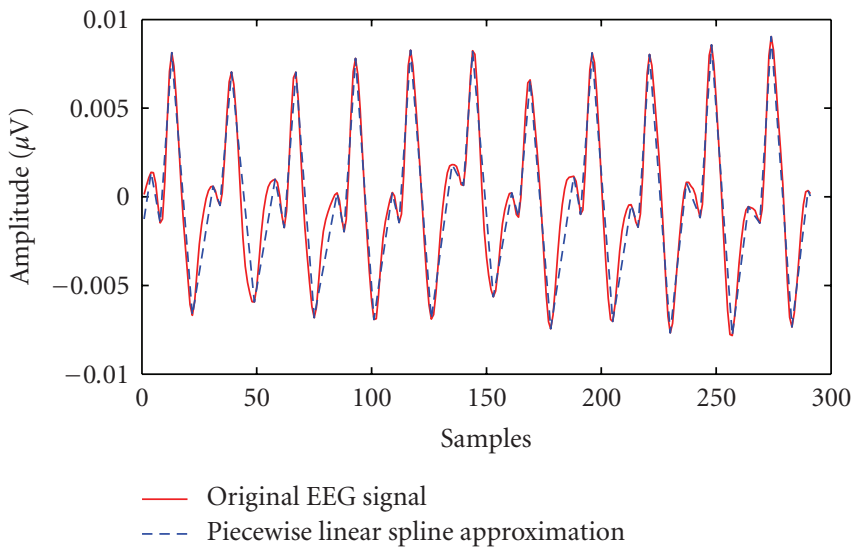

(d)

Figure 2: Examples of modelling different EEG signals with $K$-pieces nonuniform linear splines. (a) $K=37$ pieces. (b) $K=23$ pieces. (c) $K=53$ pieces. (d) $K=45$ pieces.

TABLE 1: Comparison of different approximation models for 30 epochs of EEG signals.

\begin{tabular}{lccc}
\hline Evaluation metric & Linear spline model & Quadratic spline model & Cubic spline model \\
\hline Average CC (\%) & 97.92 & 90.37 & 98.11 \\
Average RMSE & 0.0024 & 0.0059 & 0.0020 \\
Average PRD (\%) & 15.26 & 45.21 & 16.42 \\
Average MAXERR & 0.0013 & 0.027 & 0.0012 \\
\hline
\end{tabular}

where $\overline{x_{\text {orig }}}$ and $\overline{x_{\text {recon }}}$ are the mean values of the original and reconstructed signals, respectively.

In order to understand the local distortions between the original and the reconstructed signals, two metrics, the maximum error (MAXERR) and the peak amplitude related error (PARE) [19], will be computed. The maximum error metric is defined as

$$
\operatorname{MAXERR}=\max \left(x_{\text {orig }}-x_{\text {recon }}\right)
$$

and it shows how large the error is between every sample of the original and reconstructed signals. This metric should ideally be small if both signals are similar. The PARE is defined as

$$
\operatorname{PARE}(i)=\frac{\left[x_{\text {orig }}(i)-x_{\text {recon }}(i)\right]}{x_{\text {orig }}(i)} .
$$

By plotting PARE, one will be able to understand the locations and magnitudes of the errors between the original and reconstructed signals.

2.3.2. Comparison of Models. As depicted in Figure 1, a comparison between the original EEG signal and its approximations shows that the nonuniform linear spline model gives the best approximation where a CC of $99.22 \%$, with an RMSE of 0.0020 , a PRD of $13.07 \%$, and a MAXERR of 0.0012 are achieved. An evaluation of 30 sets of EEG signals in Table 1 shows that the nonuniform linear and cubic spline models best fitted the EEG signals. Since the results achieved with a linear spline model are very close to that of a cubic spline model, the nonuniform linear spline model is chosen as our EEG model to minimise computation costs.

Figure 2 illustrates the nonuniform linear spline model for different EEG signals whereby the approximations closely 


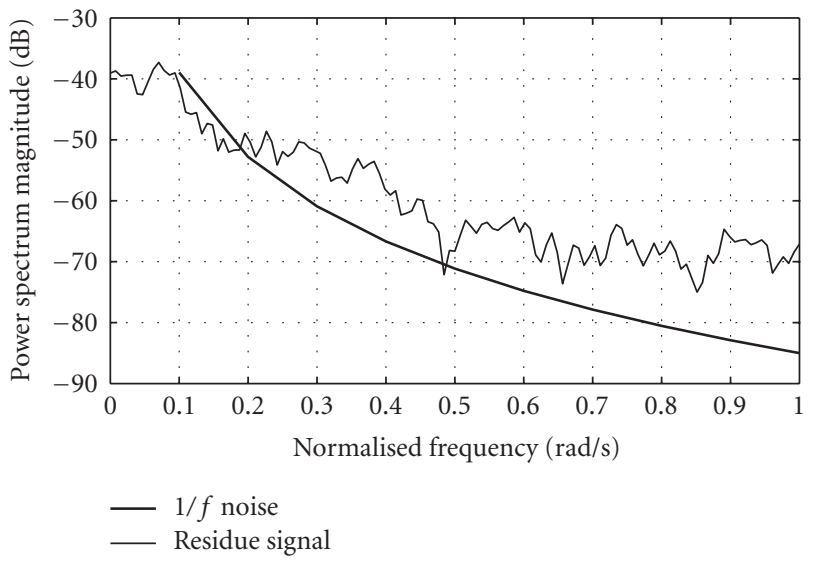

(a)

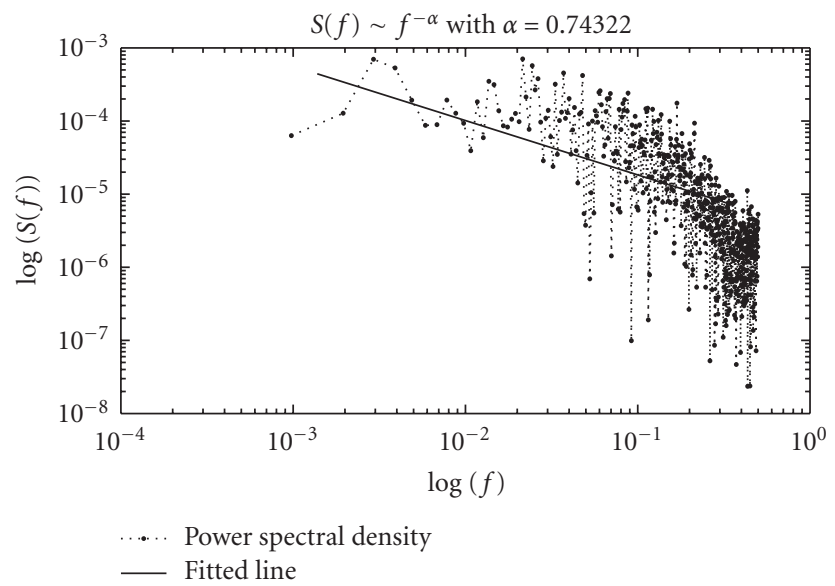

(b)

FIgURE 3: (a) The average power spectral density of the residue signal compared to that of $1 / f$ noise (b) The estimation of $\alpha$ from the log-log plot of average power spectral density versus frequency of the residue signal.

Sampling kernel

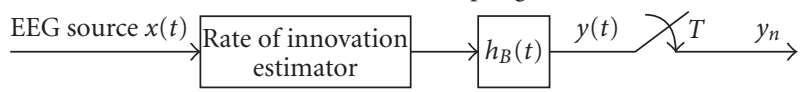

FIGURE 4: Our proposed scheme for sampling EEG signals with finite rate of innovation.

model the original signals with different $K$ pieces of linear splines. As such, we conclude that with our data, different EEG signals can be modelled with $2 K \in[350,900]$ for each 10 seconds EEG segment.

The nonuniform linear spline model makes up approximately $97 \%$ of the EEG signal. We observed that the residue signal (EEG signal-nonuniform linear splines) resembled a $1 / f$ noise signal with a power spectral density $S(f) \propto$ $1 / f^{\alpha}$. The range of this noise amplitude was found to be $[-0.003,0.002]$ and $\alpha$ was estimated to be around 0.75 by computing the slope of a fitted line onto the log-log plot of $S(f)$ versus $f$ as shown in Figures 3(a) and 3(b) [20]. Thus, our EEG signals are modelled as nonuniform linear
TABLE 2: The assumed values of $2 K$, the respective compression ratio $\mathrm{CR}$ and $C$.

\begin{tabular}{|c|c|c|c|}
\hline EEG epoch & $\begin{array}{c}\text { No. of coefficients } \\
2 K\end{array}$ & $\begin{array}{c}\text { Compression ratio } \\
\text { CR }(\%)\end{array}$ & $C$ \\
\hline P1_001 & 694 & 72.85 & 3.68 \\
\hline P1_002 & 716 & 72.03 & 3.61 \\
\hline P1_003 & 852 & 66.71 & 3.00 \\
\hline P1_004 & 640 & 75.00 & 4.00 \\
\hline P1_005 & 702 & 72.61 & 3.65 \\
\hline P1_006 & 880 & 65.63 & 2.91 \\
\hline P1_007 & 788 & 69.18 & 3.24 \\
\hline P1_008 & 784 & 69.34 & 3.26 \\
\hline P1_009 & 720 & 71.88 & 3.56 \\
\hline P1_010 & 742 & 70.98 & 3.45 \\
\hline P1_001 & 474 & 81.48 & 5.40 \\
\hline P2_002 & 582 & 77.27 & 4.40 \\
\hline P2_003 & 635 & 75.20 & 4.03 \\
\hline P2_004 & 706 & 72.42 & 3.63 \\
\hline P2_005 & 742 & 71.02 & 3.45 \\
\hline P2_006 & 490 & 80.86 & 5.23 \\
\hline P2_007 & 548 & 78.55 & 4.66 \\
\hline P2_008 & 610 & 76.17 & 4.20 \\
\hline P2_009 & 544 & 78.71 & 4.70 \\
\hline P2_010 & 888 & 65.31 & 2.89 \\
\hline P3_001 & 860 & 66.41 & 2.98 \\
\hline P3_002 & 836 & 67.34 & 3.06 \\
\hline P3_003 & 470 & 81.64 & 5.45 \\
\hline P3_004 & 682 & 73.36 & 3.75 \\
\hline P3_005 & 624 & 75.59 & 4.10 \\
\hline P3_006 & 490 & 80.86 & 5.23 \\
\hline P3_007 & 631 & 75.35 & 4.06 \\
\hline P3_008 & 755 & 70.51 & 3.39 \\
\hline P3_009 & 654 & 74.45 & 3.91 \\
\hline P3_010 & 820 & 67.97 & 3.12 \\
\hline
\end{tabular}

splines embedded in additive $1 / f$ noise. In this way, the EEG signals have been cast as signals with finite rate of innovation embedded in noise and this provides a motivation to exploit the FRI framework for compressive sampling of EEG signals.

\section{Compressive Sampling of EEG Signals with Finite Rate of Innovation}

Since the EEG signals are modelled as an FRI signal $+1 / f$ noise, we will employ methods developed in $[12,21]$ to acquire and reconstruct them. It is important that the rate of innovation of the EEG signals is known and in our case, it has to be estimated from the source signals. Let us assume that the number of pieces of linear splines needed to represent the EEG signals is given as shown in Table 2.

With these assumptions, we represent the EEG signals as $K$ pieces of nonuniform linear splines embedded in $1 / f$ noise 


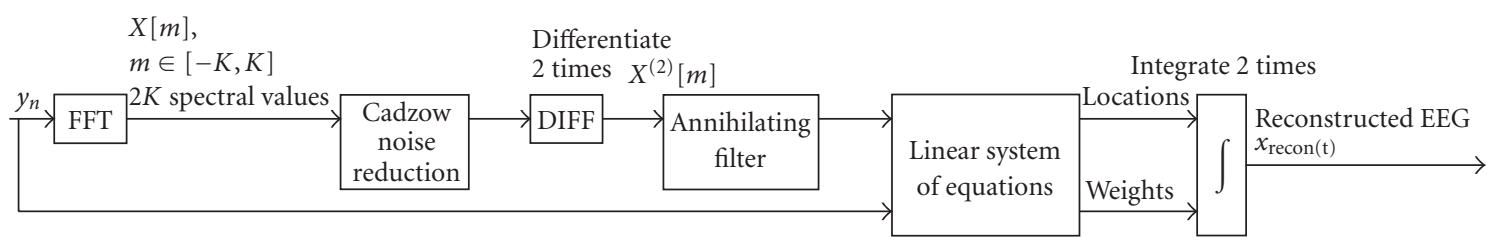

FIgURE 5: The FRI reconstruction block diagram for EEG signals.

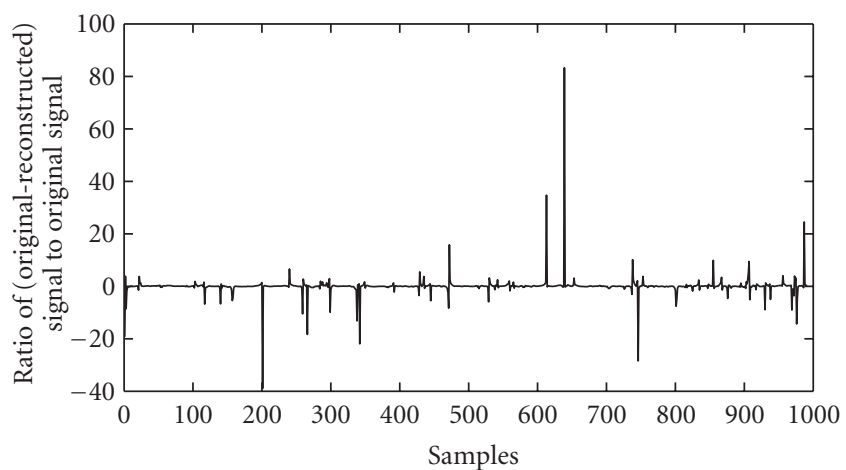

FIgURE 6: A plot of PARE for the signals shown in Figure 8. Errors between original and reconstructed samples are depicted as spikes in this plot.

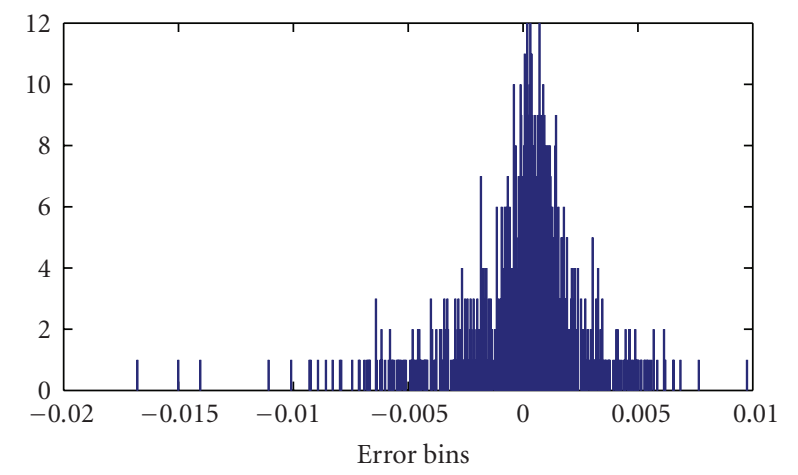

FIGURE 7: Histogram plot of the errors obtained for an epoch.

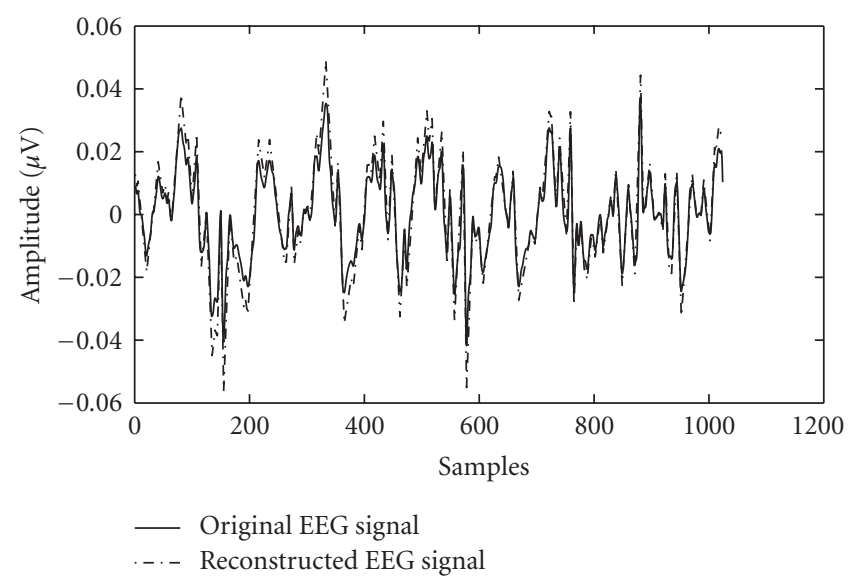

Figure 8: A comparison between the original EEG signal and its reconstruction. $\mathrm{PRD}=34.35 \%, \mathrm{CC}=98.43 \%$, $\mathrm{RMSE}=0.0046$, and MAXERR $=0.0187$.

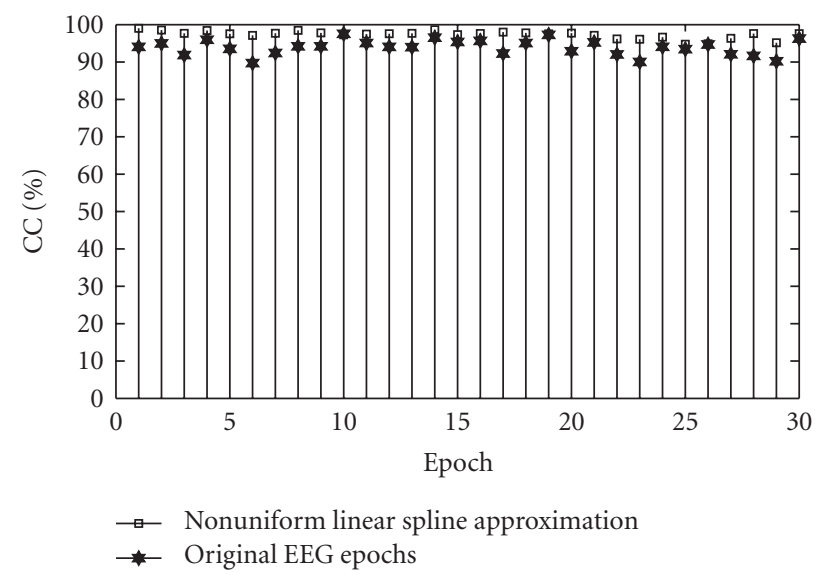

Figure 9: Comparison of the 30 epochs' CC in the event of (i) sampling the original EEG epochs and (ii) sampling their nonuniform linear spline approximations (noise free signals).

and the corresponding rate of innovation will be $\rho=2 K / \tau$ with $\tau=10$ seconds.

3.1. Our Method. Figure 4 shows our proposed EEG signal acquisition process with finite rate of innovation. Since the value of $K$ is assumed to be known, the samples of the EEG signal are obtained based on the descriptions in Section 2.2.1. Corresponding to the representation for the EEG signals, a reconstruction method is presented in Figure 5.

In order to perform the Cadzow's noise reduction, a rectangular $(L \times(L+1), L \geq K)$ Toeplitz matrix $D$ is created from the spectral values of the source signal $x(t)$ in the form

$$
D=\left(\begin{array}{cccc}
X[0] & X[-1] & \ldots & X[-L] \\
X[1] & X[0] & \ldots & X[-L+1] \\
\vdots & \vdots & \vdots & \vdots \\
X[L-1] & X[L-2] & \ldots & X[-1]
\end{array}\right) .
$$

We then perform a singular vector decomposition of the matrix $D$, and enforce rank $K$ on $D$ by choosing only $K$ most significant singular values. This is iterated until the ratio of the largest singular value of the $K$ th +1 to that of the $K$ th is smaller than a preset threshold. Thus the denoised DFT coefficients can be extracted from $D$ [21].

Since our EEG signals are modelled as nonuniform linear splines, we perform a differentiation operation twice on the denoised signals so as to reduce them into a stream of Dirac pulses. In order to find the locations and weights of the Dirac pulses, consider a filter $A[m]$ whose $z$-transform has 


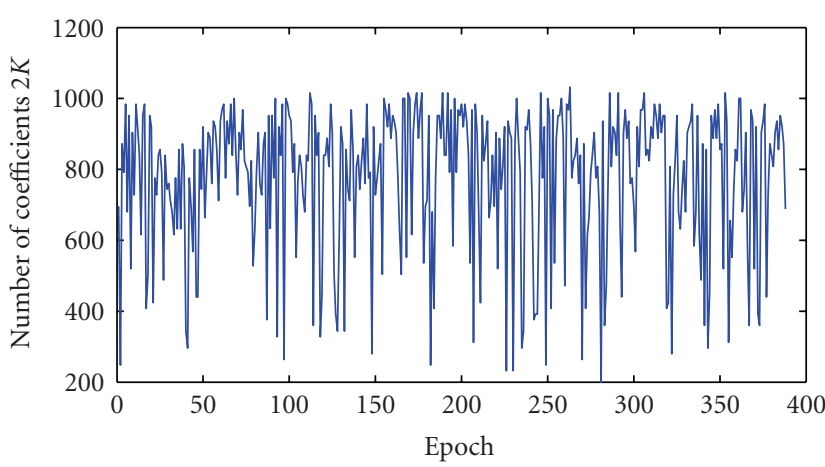

(a)

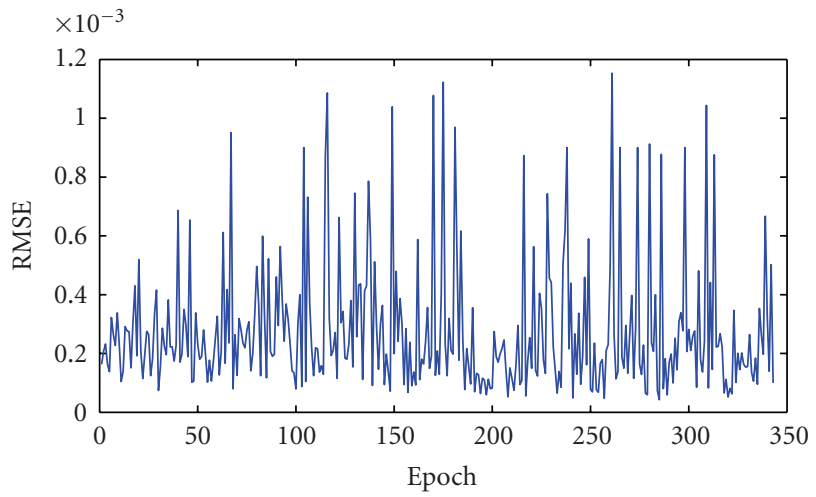

(c)

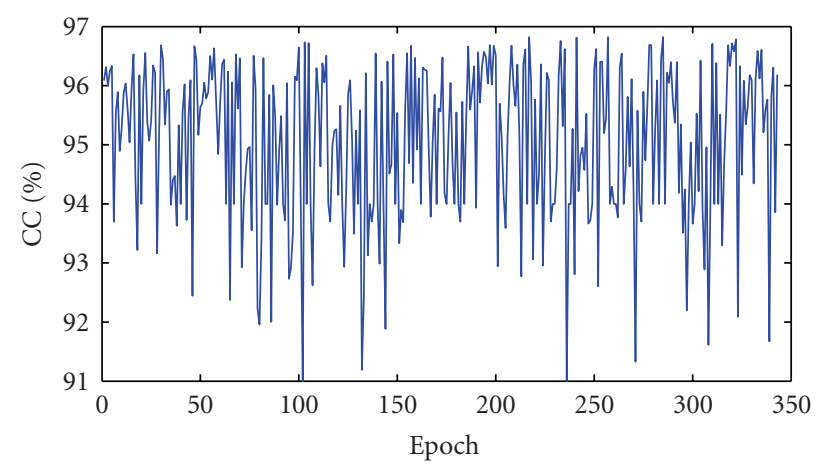

(b)

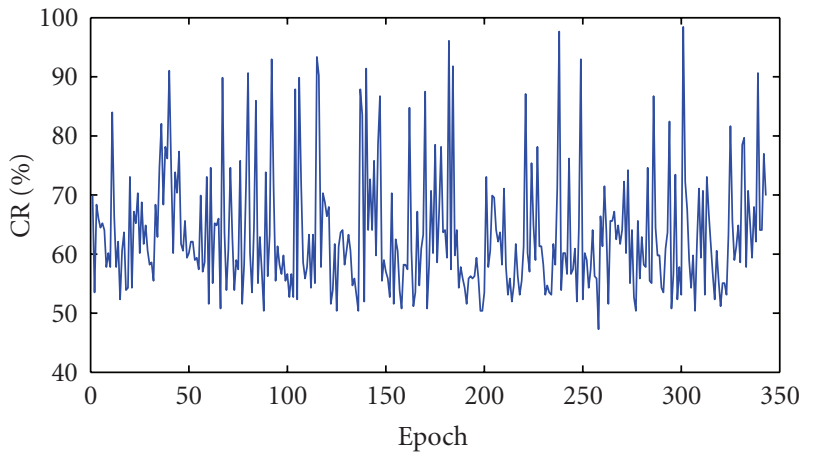

(d)

Figure 10: Variation of the evaluation metrics on 343 epochs corresponding to 3430 seconds of continuous EEG from one patient. (a) The number of coefficient $2 K$ versus epochs. (b) CC versus epochs. (c) RMSE versus epochs. (d) CR versus epochs.

$K$ zeros at $u_{k}=e^{-i\left(2 \pi t_{k} / \tau\right)}$, that is, $A(z)=\prod_{k=0}^{K-1}\left(1-u_{k} z^{-1}\right)$. Since the CTFS of the differentiated EEG signal $x^{(2)}(t)$ is a linear combination of $K$ complex exponentials $u_{k}$, it follows that $A[m]$ is an annihilating filter and satisfies the following condition:

$$
A[m] * X^{(2)}[m]=0,
$$

where

$$
X^{(2)}[m]=\left(\frac{j 2 \pi m}{\tau}\right)^{2} X[m], \quad m \in[-K, K] .
$$

The coefficients of the annihilating filter are found solving (12) which is equivalent to the following Toeplitz linear system of equations:

$$
\sum_{k=0}^{K} A[k] X^{(2)}[m-k]=0, \quad m=-K, \ldots, K
$$

Thus the locations $\left\{t_{k}\right\}_{k=0}^{K-1}$ of the Dirac pulses are given by the roots of $A(z)$. Next, the weights $\left\{c_{k}\right\}_{k=0}^{K-1}$ of the Dirac pulses are given by solving the Vandermonde system of equations given by

$$
X^{(2)}[m]=\frac{1}{\tau} \sum_{k=0}^{K-1} c_{k} e^{-j 2 \pi m t_{k} / \tau}, \quad m=0, \ldots, K-1 .
$$

Lastly, the stream of Dirac pulses is integrated twice to obtain the reconstructed EEG signals which correspond to the nonuniform linear spline approximation of the EEG signals.

\section{Results and Discussions}

In this section, we will present our results based on the performance metrics in Section 2.3.1. Comparisons to wavelet based compression techniques using discrete wavelet transform with the Daubechies and Coiflets wavelets [8] will be included in our discussions. These wavelet transforms are performed with four detailed levels and one approximation. Both the wavelet coefficients and the FRI innovation parameters are coded using Huffman coding. We also compared our results to those found in [11] in terms of normalised mean square error (NMSE), which is the ratio of mean square error of the reconstructed signals to the range of amplitudes of the signals.

We applied our method on the 3 sets of EEG signals and the results of 30 selected epochs are tabulated in Table 3.

The CC is selected as the primary evaluation metric and our results are generated with the best $\mathrm{CC}$ achievable for each EEG signal. As shown in Table 3, consistently high CC ranging from 89.71 to 97.56 is achieved for our dataset. This implies that there is a great similarity in the morphology between the original and reconstructed EEG signals. This 


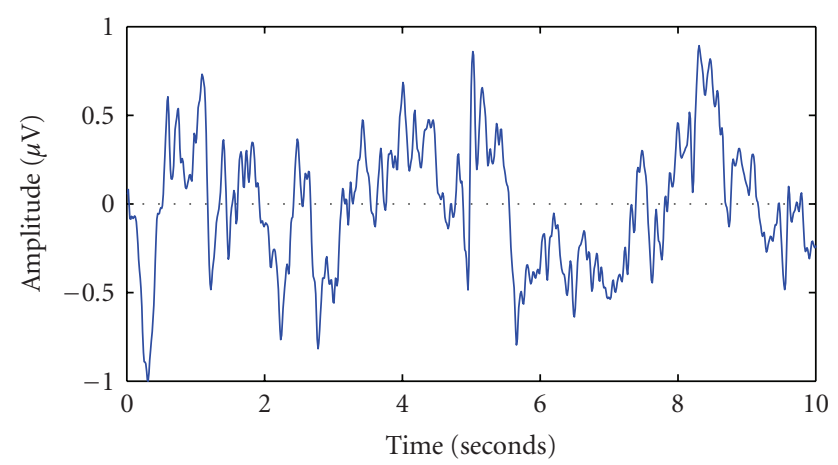

(a)

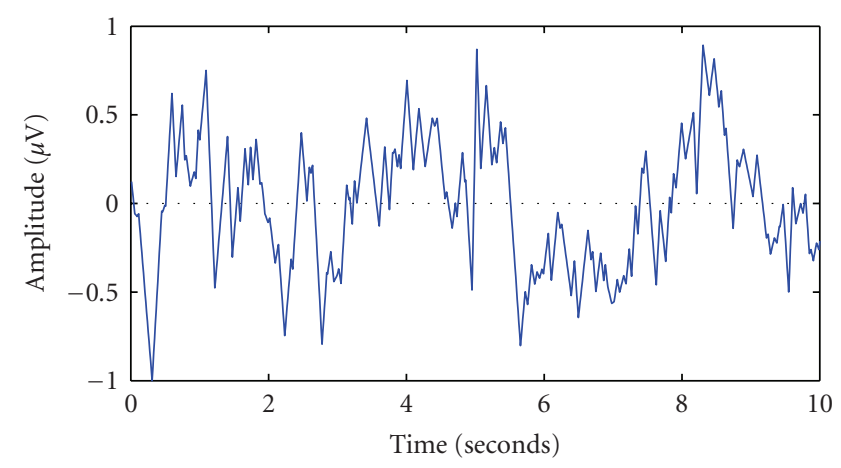

(c)

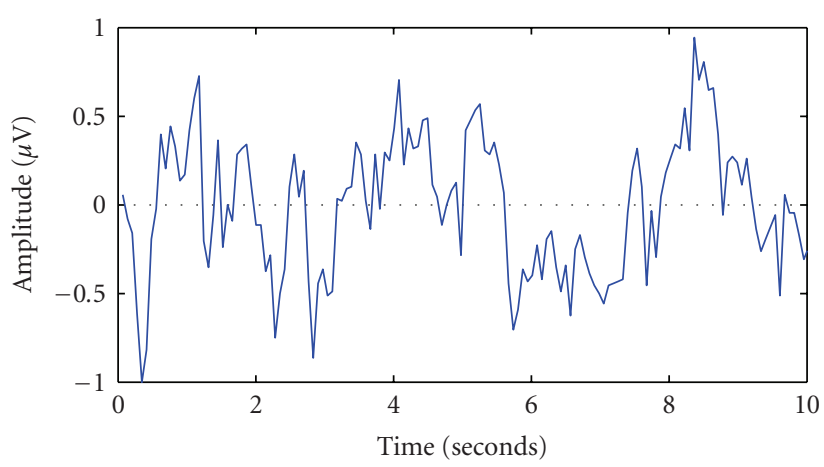

(b)

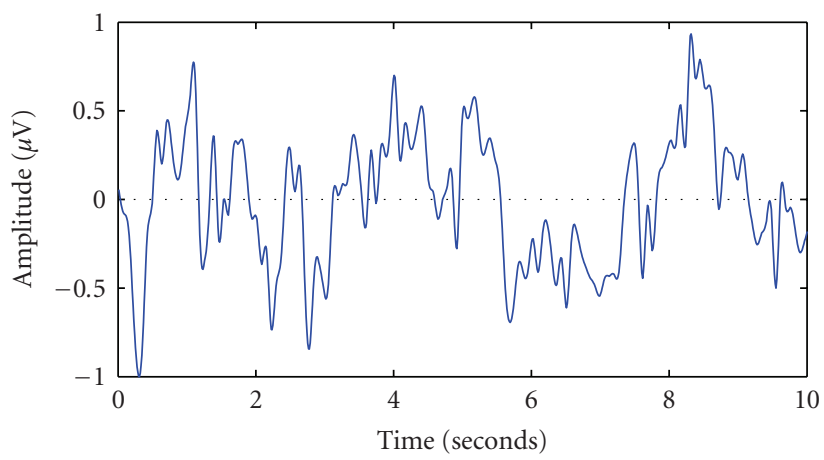

(d)

FIgure 11: Comparison of reconstruction of an EEG signal acquired by our method. (a) The original signal sampled at $256 \mathrm{~Hz}$. (b) The EEG signal acquired by our method at $47.5 \mathrm{~Hz}$ with $2 \mathrm{~K}=474$. (c) The reconstructed EEG signal at $256 \mathrm{~Hz}$ with our method CC $=97.08 \%$, $\mathrm{RMSE}=0.0099, \mathrm{PRD}=22.43 \%$, MAXERR $=0.0016$. (d) The reconstructed EEG signal using the traditional sinc interpolation method CC $=90.11 \%$, RMSE $=0.0147, \mathrm{PRD}=48.30 \%$, MAXERR $=0.428$.

result is highly desirable because such diagnostic features are extremely important and must be preserved. Our method achieves a CR ranging from $65.31 \%$ to $81.48 \%$. This is due to the morphology of the EEG signals, where some signals need more linear splines to model them compared to the others. As $K$ varies, the rate of innovation $\rho$ varies accordingly and leads to an increased or decreased number of spectral coefficients. Thus CR varies inversely as $\rho$. Table 3 also tabulates the distortions arising from our method. We obtained low RMSE and PRD, implying that our method recovers signals with some distortion. Furthermore, a MAXERR between 0.0070 and 0.0247 is obtained, suggesting that the distortions of the reconstructed signals are very small.

A typical plot of PARE and a histogram of the errors are shown in Figures 6 and 7. Errors between original samples of the signal and the corresponding reconstructed samples are amplified and shown in the PARE plot. The values of PARE are generally less than $5 \%$ of the original signal, although some PARE values are larger than $20 \%$ of the original signal. By comparing Figure 8 to Figure 6 , the PRD is relatively high at $34.35 \%$ and the CC value is $98.43 \%$. However, the differences between the original and reconstructed signals cannot be distinguished morphologically. Thus PRD alone cannot measure how well morphologies of the EEG signals are retained. The histogram of errors showed a concentration of errors in the range between -0.005 to 0.005 , and some outliers in the larger error bins, thus contributing to distortions. Although the results are satisfactory, we observed that the distortions arise from the estimation of the innovation parameters. Let us make a comparison of the CC obtained by sampling both the original EEG epochs and their nonuniform linear spline approximation (i.e., noisefree signals). If the estimation of the innovation parameters is accurate, the CC obtained should be the same. As shown in Figure 9, the CC obtained for the noise-free signal is higher than the noisy signal, due to wrong estimations of locations and weights.

In addition, we present results for sampling three 24hour recordings. Figure 10 shows how the various metrics change in a continuous EEG recording of one patient. As illustrated in Figure 10(a), the number of coefficients $2 \mathrm{~K}$ varies between 200 and 1000, thus showing that for the same patient, the innovation parameters cannot be assumed to be constant. We also noted that although $2 K$ varies in a large range, $\mathrm{CC}$ is quite consistently lying in the range of $91 \%$ to $97 \%$ as in Figure 10(b). Furthermore, the distortion in the EEG is kept very low, as depicted by Figure 10(c). Compression ratio for this recording ranges from about $50 \%$ to $99 \%$, which varies faithfully with $2 K$. Table 4 tabulates the mean CR, CC, PRD, RMSE, MAXERR, and number of coefficients for each dataset. On the average, we achieved a CR of around $62.3 \%$ with low PRD, RMSE, and MAXERR 


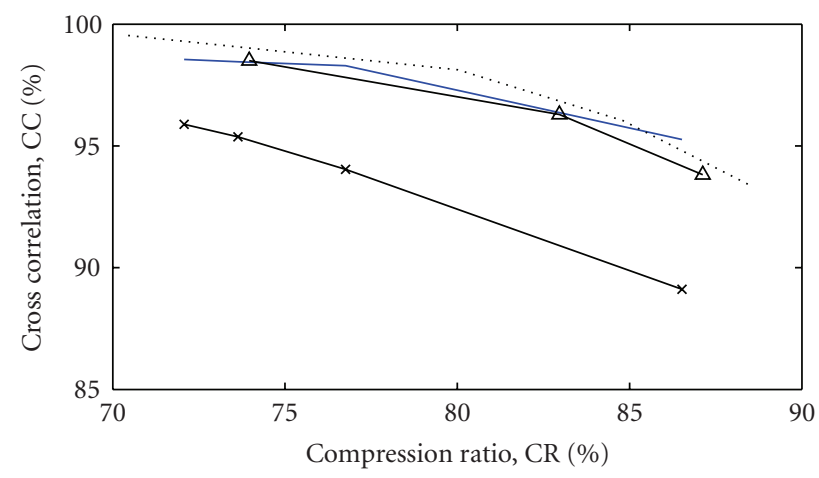

(a)

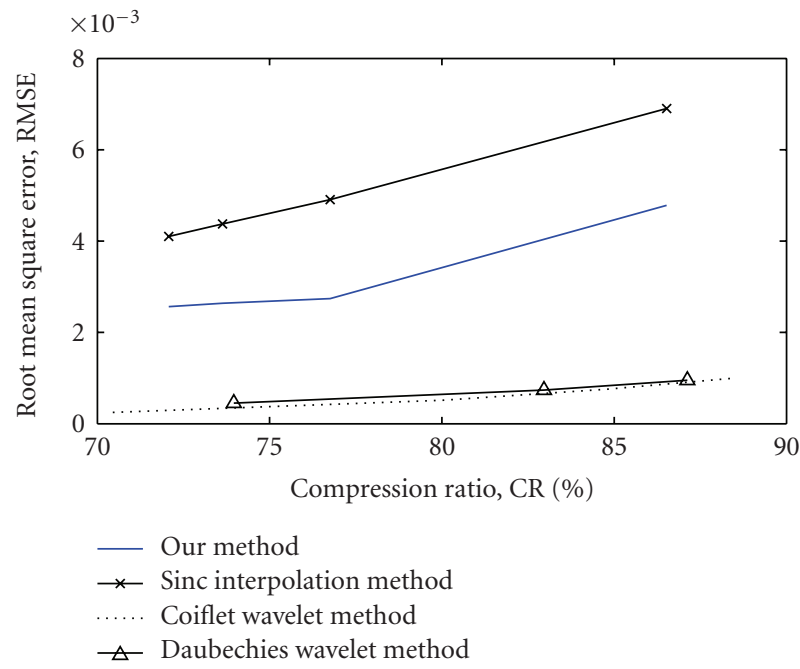

(c)

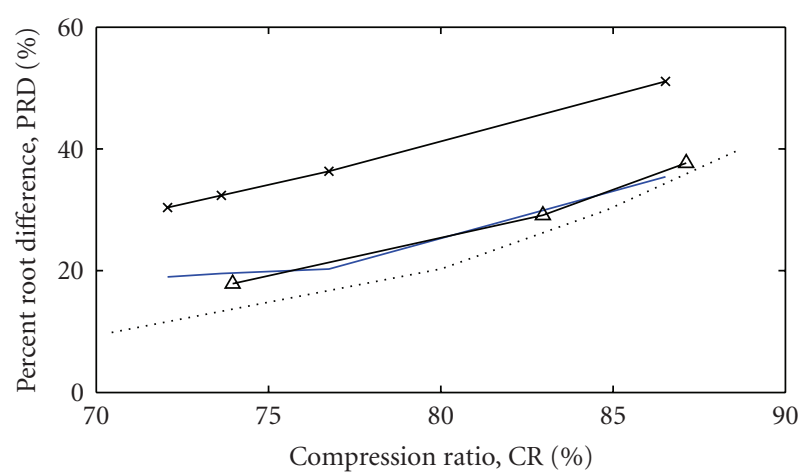

(b)

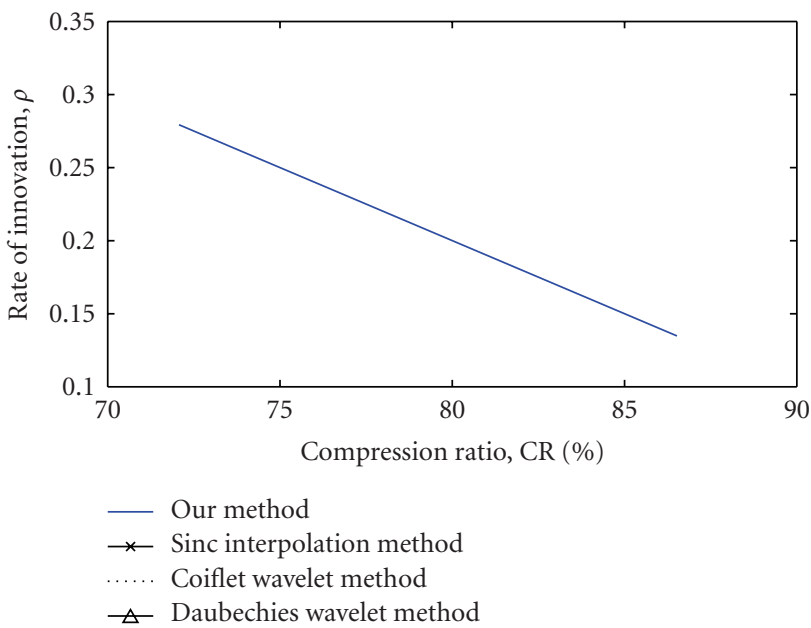

(d)

FIGURE 12: A comparison of the performance of our method with sinc interpolation, wavelet compression using Daubechies and coiflets wavelets on an EEG epoch. (a) Variation of CC with CR. (b) Variation of PRD with CR. (c) Variation of RMSE with CR. (d) Variation of $\rho$ with CR.

errors of around $33.63 \%, 0.0055$, and 0.0225 , respectively. In addition, the CC achieved is around $95.08 \%$. Based on the observations of our experiments, a minimum CC value of $90 \%$ and a maximum PRD value of $40 \%$ will maintain the morphologies of the reconstructed signals visually.

Figure 11 presents an example of the strength and uniqueness of our sampling scheme. The EEG signal's original sampling frequency is $256 \mathrm{~Hz}$ (Figure 11(a)). Our system estimated $2 K$ to be 474 and modelled the signal with 474 samples of the original signal (Figure 11(b)), which has a CR of $81.48 \%$. Effectively, we are sampling the original signal at $47.5 \mathrm{~Hz}$. We effectively reconstructed the $256 \mathrm{~Hz}$ signal as shown in Figure 11(c) with CC $=97.08 \%$, RMSE $=0.0099, \mathrm{PRD}=22.43 \%$, MAXERR $=0.0016$. As a compar ison, we reconstructed the signal in Figure 11(b) with the traditional sinc interpolation method (Figure 11(d)) with $\mathrm{CC}=90.11 \%, \mathrm{RMSE}=0.0147, \mathrm{PRD}=48.30 \%$, MAXERR $=0.428$. Clearly, we are able to represent EEG signals with a low number of samples and reconstruct them with high fidelity.

Figure 12 shows therelation between CC, RMSE, PRD, and $\rho$ with CR, respectively. The CR is inversely proportional to the rate of innovation, as shown in the earlier discussion. In order to achieve a high CC, CR has to be compromised. Similarly, as we increase CR, the error involved such as RMSE and PRD will increase together, though not in a linear form. A comparison is made with traditional sinc interpolation and the performance of our method is superior since an interpolation process is unable to faithfully reconstruct signals acquired at a low-sampling rate into one of a higher sampling rate. Next we compared our results with that of compressing the EEG signals using wavelet compression methods. As illustrated, our method achieves comparable results in terms of CC and PRD although the RMSE achieved by wavelet methods is slightly better. Since RMSE only indicates how much error is incurred in the reconstruction without reference to the morphology of the signals, our results do not indicate that our reconstructed signals differ largely from the original signals morphologically. Furthermore, our method entails a less costly low-rate sampling device and does not waste precious computational resources collecting extra data only to discard them subsequently.

Figure 13 illustrates a comparison between an original and a reconstructed EEG signal with the $2 K=1184$ 
TABLE 3: Performance of our method: CC, RMSE, PRD, and MAXERR.

\begin{tabular}{|c|c|c|c|c|}
\hline EEG & Cross correlation CC (\%) & Root mean square error RMSE & Percent root difference PRD (\%) & Maximum error MAXERR \\
\hline P1_001 & 94.05 & 0.0067 & 39.35 & 0.0235 \\
\hline P1_002 & 94.98 & 0.0082 & 32.37 & 0.0247 \\
\hline P1_003 & 91.87 & 0.0051 & 30.43 & 0.0212 \\
\hline P1_004 & 95.95 & 0.0024 & 34.90 & 0.0187 \\
\hline P1_005 & 93.52 & 0.0122 & 40.05 & 0.0204 \\
\hline P1_006 & 89.71 & 0.0066 & 51.37 & 0.0231 \\
\hline P1_007 & 92.42 & 0.0183 & 49.37 & 0.0213 \\
\hline P1_008 & 94.20 & 0.0091 & 35.43 & 0.0220 \\
\hline P1_009 & 94.20 & 0.0081 & 38.54 & 0.0186 \\
\hline P1_010 & 97.56 & 0.0039 & 23.05 & 0.0070 \\
\hline P2_001 & 95.08 & 0.0099 & 39.35 & 0.0102 \\
\hline P2_002 & 94.05 & 0.0055 & 39.78 & 0.0151 \\
\hline P2_003 & 93.98 & 0.0070 & 31.09 & 0.0211 \\
\hline P2_004 & 96.51 & 0.0069 & 31.52 & 0.0098 \\
\hline P2_005 & 95.36 & 0.0100 & 34.85 & 0.0178 \\
\hline P2_006 & 95.64 & 0.0063 & 30.83 & 0.0123 \\
\hline P2_007 & 92.28 & 0.0007 & 34.31 & 0.0210 \\
\hline P2_008 & 95.07 & 0.0008 & 36.31 & 0.0090 \\
\hline P2_009 & 97.26 & 0.0046 & 27.45 & 0.0012 \\
\hline P2_010 & 92.88 & 0.0096 & 43.52 & 0.0244 \\
\hline P3_001 & 95.25 & 0.0052 & 30.19 & 0.0231 \\
\hline P3_002 & 92.02 & 0.0031 & 32.11 & 0.0235 \\
\hline P3_003 & 90.03 & 0.0011 & 33.17 & 0.0209 \\
\hline P3_004 & 94.08 & 0.0032 & 33.27 & 0.0125 \\
\hline P3_005 & 93.44 & 0.0024 & 32.75 & 0.0102 \\
\hline P3_006 & 94.69 & 0.0011 & 37.07 & 0.0196 \\
\hline P3_007 & 92.07 & 0.0019 & 32.70 & 0.0180 \\
\hline P3_008 & 91.63 & 0.0012 & 28.33 & 0.0219 \\
\hline P3_009 & 90.20 & 0.0039 & 36.37 & 0.0243 \\
\hline P3_010 & 96.27 & 0.0025 & 29.95 & 0.0023 \\
\hline
\end{tabular}

TABle 4: Performance of our method on the 3 EEG datasets.

\begin{tabular}{lcccc}
\hline EEG & Patient 1 & Patient 2 & Patient 3 & Average \\
\hline Mean no. of coefficients & 941 & 982 & 973 & 965 \\
Mean CR (\%) & 63.24 & 61.53 & 61.98 & 62.3 \\
Mean CC (\%) & 94.30 & 95.32 & 95.63 & 95.08 \\
Mean PRD (\%) & 34.89 & 32.72 & 33.29 & 33.63 \\
Mean RMSE & 0.0080 & 0.0061 & 0.0025 & 0.0055 \\
Mean MAXERR & 0.0201 & 0.0302 & 0.0171 & 0.0225 \\
\hline
\end{tabular}

coefficients, we reconstructed the signal and achieved CR $=81.48 \%, \mathrm{CC}=97.52 \%, \mathrm{RMSE}=0.0044, \mathrm{PRD}=24.55 \%$, and MAXERR $=0.002$. Furthermore, the NMSE of the reconstructed signal is in the range of $5 \times 10^{-9}$ to $7.2 \times 10^{-7}$ as opposed to 0.01 achieved by the method in [11]. A zoomedin view in Figure 14 confirms that the morphology of the original signal is conserved in the reconstruction.
Lastly we will discuss about the computational costs of our scheme. With reference to Figure 5, the computational complexity can be estimated as follows.

(i) Compute the DFT to obtain the Fourier series coefficients $\in[-K, K]: O(K \log K)$.

(ii) Denoise: $O\left(K^{3}\right)$.

(iii) Differentiate the denoised signal: $O(K)$.

(iv) Solve a Toeplitz system of equation of size $K$ by $K$ to get $A(z): O\left(K^{2}\right)$.

(v) Find the roots of $A(z)$ by factorization, to get $t_{k}: O\left(K^{3}\right)$.

(vi) Solve a Vandermonde system of equation of size $K$ by $K$ to get $c_{k}: O\left(K^{2}\right)$.

(vii) Integrate the $K$ Dirac pulses- $O(J)$

Hence effectively, the computational costs involved is $O\left(K^{3}+J\right)$. For our dataset, the average time required to 


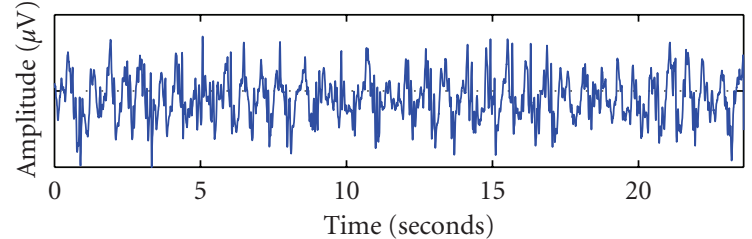

(a)

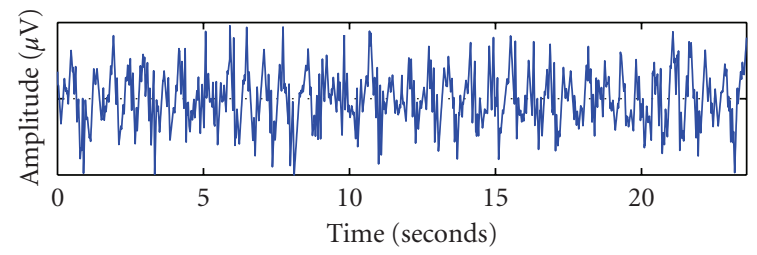

(b)

FIGURE 13: A comparison between (a) an original EEG signal and (b) the reconstructed EEG signal. $2 K=1184, \mathrm{CR}=81.48 \%, \mathrm{CC}=$ $97.52 \%, \mathrm{RMSE}=0.0044, \mathrm{PRD}=24.55 \%$, MAXERR $=0.0022$.

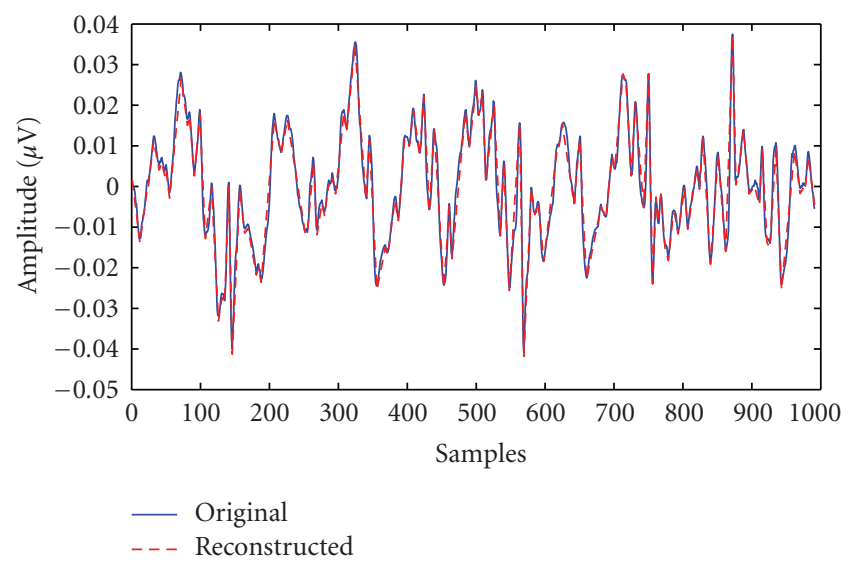

Figure 14: A zoom-in view of the comparison of original and reconstructed EEGs in Figure 13.

sample and reconstruct a 10-second epoch is 5.009 seconds on an Intel Core2 Duo $2.50 \mathrm{GHz}$ system with $4 \mathrm{~GB}$ RAM. This computational time can be improved by employing fast algorithms on dedicated digital signal processors to achieve a realtime EEG signal acquisition and display.

\section{Conclusions}

We proposed an approach to compress EEG signals at source based on the finite rate of innovation sampling theory. Unlike traditional compression methods which acquire many data samples and later discard redundant ones, our proposed method relies on acquiring a small set of data from the original signal based on the signal's rate of innovation and then reconstructing the signal with high resolution. Even though a small set of data is obtained, our method retains the morphologies of the EEG signals. It yielded promising results such as good cross correlation and low distortions at a low computational cost. In this way, we achieve computational savings which can be utilised in other more important signal processing stages. Moderate $C$ ratios are obtained for some epochs, leading to a moderate compression ratio. Furthermore, it is observed that $K$ changes depending on the state of the EEG, thus leading to a variable rate of innovation. Valuable information such as the occurrences of EEG abnormalities can be extracted through tracking the changes in the rate of innovation across the EEG. As such, the advantage of our compression method lies in the ability to compress EEG signals and track changes across EEG states concurrently. Although the accuracy of the estimated $K$ affects the entire scheme, as discussed in [21], it can be estimated from the rank of a Toeplitz matrix. However, more research is needed to determine the correct duration of EEG signals to yield optimal $K$ values based on certain evaluation metrics such as CR, CC, PRD, or MAXERR.

We will continue our work to minimise the local errors caused by outliers and to include adaptive rate of innovation to cater to the changing states of EEG signals. Finally we will investigate how EEG signals can be source compressed with finite rate of innovation in real time.

\section{References}

[1] H. Berger, "Über das Elektrenkephalogramm des Menschen," Archiv für Psychiatrie und Nervenkrankheiten, vol. 87, no. 1, pp. 527-570, 1929.

[2] D. Hirtz, D. J. Thurman, K. Gwinn-Hardy, M. Mohamed, A. R. Chaudhuri, and R. Zalutsky, "How common are the “common" neurologic disorders?" Neurology, vol. 68, no. 5, pp. 326-337, 2007.

[3] G. Antoniol and P. Tonella, "EEG data compression techniques," IEEE Transactions on Biomedical Engineering, vol. 44, no. 2, pp. 105-114, 1997.

[4] H. Gürkan, U. Guz, and B. S. Yarman, "EEG signal compression based on classified signature and envelope vector sets," International Journal of Circuit Theory and Applications, vol. 37, no. 2, pp. 351-363, 2009.

[5] N. Memon, X. Kong, and J. Cinkler, "Context-based lossless and near-lossless compression of EEG signals," IEEE Transactions on Information Technology in Biomedicine, vol. 3, no. 3, pp. 231-238, 1999.

[6] T. Madan, R. Agarwal, and M. N. S. Swamy, "Compression of long-term EEG using power spectral density," in Proceedings of the 26th Annual International Conference of the IEEE Engineering in Medicine and Biology (EMBS '04), pp. 180-183, September 2004.

[7] Y. Wongsawat, S. Oraintara, T. Tanaka, and K. R. Rao, "Lossless multi-channel EEG compression," in Proceedings of IEEE International Symposium on Circuits and Systems (ISCAS '06), pp. 1611-1614, Island of Kos, Greece, May 2006.

[8] M. Nielsen, E. N. Kamavuako, M. M. Andersen, M.-F. Lucas, and D. Farina, "Optimal wavelets for biomedical signal compression," Medical and Biological Engineering and Computing, vol. 44, no. 7, pp. 561-568, 2006.

[9] N. Sriraam, "Neural network based near-lossless compression of EEG signals with non uniform quantization," in Proceedings of the Annual International Conference of the IEEE Engineering in Medicine and Biology Society (EMBS '07), pp. 3236-3240, Lyon, France, August 2007. 
[10] R. G. Baraniuk, E. Candes, R. Nowak, and M. Vetterli, “Compressive sampling," IEEE Signal Processing Magazine, vol. 25, no. 2, pp. 12-13, 2008.

[11] S. Aviyente, "Compressed sensing framework for EEG compression," in Proceedings of IEEE Workshop on Statistical Signal Processing, pp. 181-184, Madison, Wis, USA, August 2007.

[12] M. Vetterli, P. Marziliano, and T. Blu, "Sampling signals with finite rate of innovation," IEEE Transactions on Signal Processing, vol. 50, no. 6, pp. 1417-1428, 2002.

[13] Y. Hao, P. Marziliano, M. Vetterli, and T. Blu, "Compression of ECG as a signal with finite rate of innovation," in Proceedings of the 27th Annual International Conference of the IEEE Engineering in Medicine and Biology (EMBS '05), vol. 7, pp. 7564-7567, Shanghai, China, September 2005.

[14] K.-K. Poh and P. Marziliano, "Compression of neonatal EEG seizure signals with finite rate of innovation," in Proceedings of IEEE International Conference on Acoustics, Speech and Signal Processing (ICASSP '08), pp. 433-436, Las Vegas, Nev, USA, March-April 2008.

[15] D. Kandaswamy, T. Blu, and D. Van De Ville, "Analytic sensing: direct recovery of point sources from planar Cauchy boundary measurements," in Wavelets XII, vol. 6701 of Proceedings of SPIE, San Diego, Calif, USA, August 2007.

[16] EEG time series Database, https://epilepsy.uni-freiburg.de/ freiburg-seizure-prediction-project/eeg-database.

[17] M. Unser, "Splines: a perfect fit for signal and image processing," IEEE Signal Processing Magazine, vol. 16, no. 6, pp. 22-38, 1999.

[18] M. S. Keshner, "1/f noise," Proceedings of the IEEE, vol. 70, no. 3, pp. 212-218, 1982.

[19] L. J. Hadjileontiadis, Biosignals and Compression Standards, Springer, Berlin, Germany, 2006.

[20] P. E. McSharry and B. D. Malamud, "Quantifying selfsimilarity in cardiac inter-beat interval time series," Computers in Cardiology, vol. 32, pp. 459-462, 2005.

[21] T. Blu, P.-L. Dragotti, M. Vetterli, P. Marziliano, and L. Coulot, "Sparse sampling of signal innovations: theory, algorithms, and performance bounds," IEEE Signal Processing Magazine, vol. 25, no. 2, pp. 31-40, 2008. 